\title{
MATHEMATICAL APPARATUS USED IN “METHOD OF MODELS" FOR THE STUDY OF FOUR-BAR LINKAGE MECHANISM (RRRR)
}

\author{
Roumen Roussev \\ Faculty of Technics and Technologies of Yambol, Trakia University of Stara Zagora, Bulgaria \\ Faculty of Engineering, South-West University "Neofit Rilski", Blagoevgrad, Bulgaria \\ e-mail: roussev_r@abv.bg
}

\begin{abstract}
The method of models" is a graphical method for determining kinematic characteristics of links and points of polycontoured mechanism. For this purpose must find their images in the "secondary" and "tertiary" mechanism's model. In present paper mathematical approach, replacing graphical work with analytical calculations is proposed, to analyze four-bar linkage mechanism.
\end{abstract}

Key words: method of models, kinematic characteristics, four-bar linkage, mathematical apparatus.

\section{INTRODUCTION}

Method of models is proposed by Piperkov [1] and developed by Tenchev [2]. Trough it can be determined kinematic characteristics of random links and points of polycontoured mechanism without calculate it for previous ones. Only need to draw a mechanism in the desired position, which is assumed to be "primary model". This is displacement function for selected value of the input parameter. Graphic representation of "secondary model" / for determining velocities/ and "tertiary model" / for determining accelerations/ of mechanism is carried out according to certain rules. Advantage of this method is that it can obtain correct results for trigonometric functions for angles $\varphi=k . \pi / 2(k=1,2, \ldots)$. The indefiniteness of arcos functions is being avoided. The disadvantage of this method is great graphics work. Usually this method is used to analyze 12 positions of the mechanism or to clarify "conflict" positions obtained by analytical methods.

Typically polycontoured mechanisms are constructed from elementary four-linkage mechanisms.

In this work mathematical apparatus is available for determining the coordinates of the characteristic points of the four-bar linkage mechanism in its models without graphic representation. This enables research to be conducted for any number of positions of the mechanism.

\section{NATURE OF “METHOD OF MODELS” FOR FOUR-BAR LINKAGE MECHANISM.}

The mechanism is built for the desired position. The centre of the model has been selected, for RRRR it is the axis of rotation of input link - point $O$ (picture below).

\subsection{Secondary model}

$1^{\prime}$. The main link of the mechanism 1 and its secondary image $1^{\prime}$ coincide, i.e. $1 \equiv 1^{\prime}$ and $A \equiv$ $A^{\prime}$.

$2^{\prime}$. If the secondary image $A^{\prime}$ of point $A$ of the rocker is known, then secondary image $B^{\prime}$ of point $B$ is located on the line which passes through $A^{\prime}$ and is parallel to line $A B$. Points $A^{\prime} B^{\prime}$ determine the position of the secondary image of the rocker in secondary model of the 


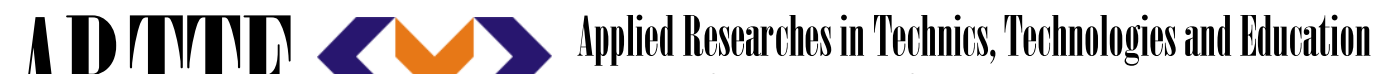 Journal of the Faculty of Technics and Technologies, Trakia University https://sites.google.com/a/trakia-uni.bg/artte/}

mechanism. The velocity $V_{B}=\omega_{1} . O B^{\prime}$ of point $B$ is perpendicular to the segment $O B^{\prime}$ and is with direction corresponding of $\omega_{1}$ relative to the center $O$. Angular velocity of the rocker $A B$ is: $\omega_{A B}=\frac{A^{\prime} B^{\prime}}{A B}$. $\omega_{1}$. If directed segments $A B$ and $A^{\prime} B^{\prime}$ are unidirectional, then angular velocity $\omega_{A B}$ has the same direction as $\omega_{1}$. If they are not, angular velocity $\omega_{A B}$ has opposite direction of $\omega_{1}$.

Secondary image $M$ of third point $M$ on the rocker $A B$ is obtained by triangle $A^{\prime} B^{\prime} M^{\prime}$, which is similar and equally oriented with triangle $A B M$. Velocity of point $M: V_{M}=\omega_{1} . O M^{\prime}$ is perpendicular to the segment $O M^{\prime}$ with direction corresponding of $\omega_{1}$ relative to the center $O$. $3^{\prime}$. Secondary image $C^{\prime}$ of point $C$ of the rocker coincide with center $O$, and secondary image $B^{\prime}$ of point $B$ lies on line that passes through the center $O$, parallel to the line $B C$. Points $C^{\prime}$ and $B^{\prime}$ determine the position of secondary image of the rocker $B C$ in the secondary model of the mechanism. The magnitude of the angular velocity of the rocker $B C$ is: $\omega_{B C}=\frac{C^{\prime} B^{\prime}}{C B} \cdot \omega_{1}$. If directed segments $C B$ and $C^{\prime} B^{\prime}$ are unidirectional, then angular velocity $\omega_{B C}$ has direction of $\omega_{1}$. If they are not, angular velocity $\omega_{B C}$ has opposite direction of $\omega_{1}$.

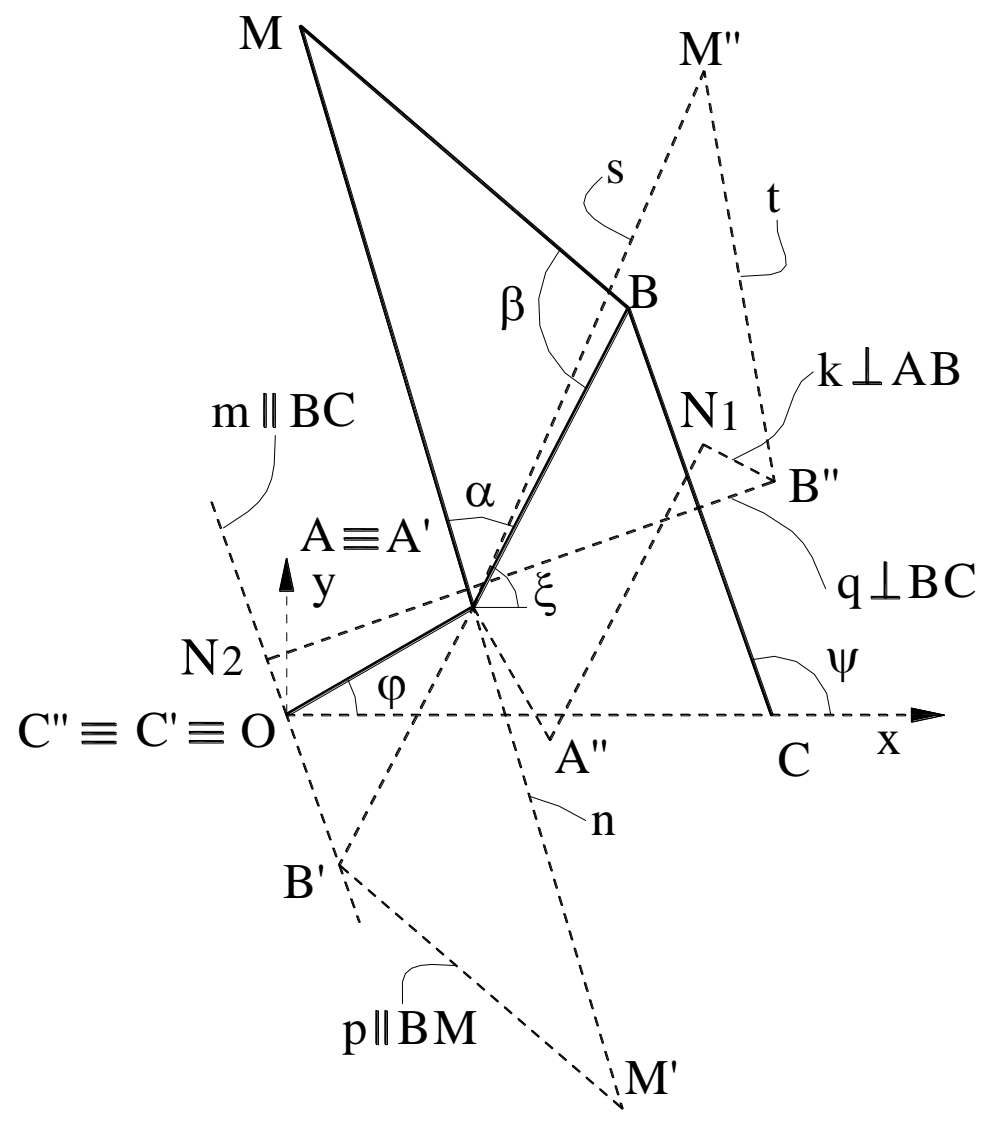

Figure 1.

Graphic constructions for Secondary and Tertiary model 


\section{ARTTE $Y$}

Ipplied Resseirl ches in Technics, Technologies and Bductation

Journal of the Faculty of Technics and Technologies, Trakia University https://sites.google.com/a/trakia-uni.bg/artte/

\subsection{Tertiary model}

1". If input link 1 has angular velocity $\omega_{1}=$ const $\left(\varepsilon_{1}=0\right)$, then link 1 and its tertiary image 1 " coincide i.e. $1 \equiv 1^{\prime \prime}$ and $A \equiv A^{\prime \prime}$. Points $O$ and $A^{\prime \prime}$ determine tertiary image of primary link in the tertiary model of the mechanism. If the acceleration of the primary link 1 is $\varepsilon_{1} \neq 0$, tertiary image $A^{\prime \prime}$ of point $A$ is shifted at a distance $A A^{\prime \prime}=\frac{\varepsilon_{1}}{\omega_{1}^{2}} \cdot O A$. Directed segment $A A^{\prime \prime}$ is perpendicular to the segment $O A$ with direction opposite to acceleration $\varepsilon_{1}$.

2 2. If tertiary image $A$ " of point $A$ on the rocker is known, the tertiary image $B$ " of point $B$ lies on line, perpendicular to segment $A B$ at a distance $A^{\prime \prime} N_{1}$ of image $A^{\prime \prime}$. Segment $A^{\prime \prime} N_{1}$ is defined as $A^{\prime \prime} N_{1}=\frac{\left(A^{\prime} B^{\prime}\right)^{2}}{A B}$. The direction from $A^{\prime \prime}$ to $N_{1}$ corresponds to the direction from $A$ to $B$. Points $A$ " and $B$ " determine position of tertiary image of the rocker in the tertiary model of the mechanism. Acceleration $\overrightarrow{a_{B}}$ in tertiary model is directed from $B$ " to $O$ with magnitude $a_{B}=\omega_{1}^{2} . O B^{\prime \prime}$. If the segment $B^{\prime \prime} N_{1}$ is considered as a vector $\overrightarrow{B^{\prime \prime} N_{1}}$ and observes rotation of segment $A^{\prime \prime} B$ " on it about point $A$ ", then it is the direction of angular acceleration $\varepsilon_{A B}$ of the rocker. The magnitude of this acceleration is $\varepsilon_{A B}=\frac{B^{\prime \prime} N_{1}}{A B} \cdot \omega_{1}^{2}$.

The tertiary image $M$ " of third point $M$ on the rocker is obtained by triangle $A^{\prime \prime} B{ }^{\prime \prime} M$ ", which is similar and equally oriented with triangle $A B M$. Acceleration $\overrightarrow{a_{M}}$ in tertiary model has direction from $M^{\prime \prime}$ to $O$ with magnitude $a_{M}=\omega_{1}^{2} . O M^{\prime \prime}$.

3". The tertiary image $C^{\prime \prime}$ of point $C$ of the rocker coincide with center $O$, and tertiary image $B^{\prime \prime}$ of point $B$ lies on line which is perpendicular to the segment $B C$ at a distance $C^{\prime \prime} N_{2}$ from the center $C^{\prime \prime} \equiv O$. The magnitude of $C^{\prime \prime} N_{2}$ is determined by the formula $C^{\prime \prime} N_{2}=\frac{\left(C^{\prime} B^{\prime}\right)^{2}}{C B}$. The direction from $C$ " to $N_{2}$ corresponds to the direction from $C$ to $B$. Points $C$ " and $B^{\prime \prime}$ determine the position of tertiary image of the crank in the tertiary model of the mechanism. If the segment $C^{\prime \prime} N_{2}$ is considered as a vector $\overrightarrow{C^{\prime \prime} N_{2}}$ and track rotation of segment $C$ " $B$ " on it about point $C^{\prime \prime}$, then it is the direction of angular velocity $\varepsilon_{B C}$ of the crank with magnitude $\varepsilon_{C B}=\frac{C^{\prime \prime} N_{2}}{C B} \cdot \omega_{1}^{2}$.

\subsection{Graphic constructions}

a. Primary model: Mechanism is built in desired position in scale 1: 1 (picture above). Coordinate axis $O x$ is assumed to coincide with the segment $O C$.

b. Secondary model: through the point $O$ line $m \| B C$ is plotted, segment $A B$ is extended to its intersection with $m$; intersection point is p. $B^{\prime}$; Line $p \| B M$ is plotted through point $B^{\prime}$, through point $A$ line $n$ (extended segment $A M$ ); intersection point between $m$ and $p$ is point M'.

c. Tertiary model: position of point $A$ " is located; position of point $N_{1}$ and $N_{2}$ is located and through them lines $k \perp A B$ and $q \perp B C$; is plotted; its intersection point is $\mathrm{B}^{\prime \prime}$; lines $s$ and $t$ are built so that $\triangle A " B " M "$ is similar to $\triangle A B M$.

IRI'IIE Vol. 4, No. 3, 2016 ISSN 1314-8788 (print), ISSN 1314-8796 (online), doi: 10.15547/artte.2016.03.004 


\section{IRITIE \\ Ipplied Researrohes in Technics, Technologies and Bducition Journal of the Faculty of Technics and Technologies, Trakia University https://sites.google.com/a/trakia-uni.bg/artte/}

\section{MATHEMATICAL APPARATUS FOR DETERMINING CHARACTERISTIC POINTS OF MECHANISM}

From the "primary model" the coordinates of characteristic points is determined:

Point A: $x_{A}=O A \cdot \cos \varphi y_{A}=O A \cdot \sin \varphi$

Point B: $x_{B}=O C+C B \cdot \cos \psi \quad y_{B}=C B \cdot \sin \psi$

Point C: $x_{c}=O C \quad y_{c}=0$

The angle $\xi$ between rocker $A B$ and coordinate axis $O x$ is determine from primary model of mechanism or $\cos \xi=\frac{x_{B}-x_{A}}{A B}$.

\subsection{Secondary model}

- Coordinates of p. $A^{\prime}: x_{A^{\prime}}=x_{A} \quad y_{A^{\prime}}=y_{A} ;$ coordinates of p. $C^{\prime}: x_{C^{\prime}}=0 ; y_{C^{\prime}}=0$

- Slopes of the lines, determined by segments: $O A \rightarrow K a=\frac{y_{A}}{x_{A}} ; C B \rightarrow K b=\frac{y_{B}}{x_{B}-O C}$

$A B \rightarrow K_{A B}=\frac{y_{B}-y_{A}}{x_{B}-x_{A}} ; A M \rightarrow K_{A M}=\frac{y_{M}-y_{A}}{x_{M}-x_{A}} ; B M \rightarrow K_{B M}=\frac{y_{M}-y_{B}}{x_{M}-x_{B}}$

- Coordinates of p. $\mathrm{P}$ (intersection point between line defined by segment $A B$ and coordinate axis $O x)$

$x_{P}=x_{A}-\frac{y_{A}}{K_{A B}}$

- Length of the segment $O B^{\prime}: O B^{\prime}=O C \cdot \frac{x_{P}}{x_{P}-x_{C}}$

- Coordinate of the p. $B^{\prime}: x_{B^{\prime}}=O B^{\prime} \cdot \cos \psi ; y_{B^{\prime}}=O B^{\prime} \cdot \sin \psi$

- Length of the segment $A^{\prime} B^{\prime}: A^{\prime} B^{\prime}=\sqrt{\left(x_{A^{\prime}}-x_{B^{\prime}}\right)^{2}+\left(y_{A^{\prime}}-y_{B^{\prime}}\right)^{2}}$

- Slope of the line, determined by segment $A^{\prime} M^{\prime}$ is the same as $A M$.

- Slope of the line, determined by segment $B^{\prime} M^{\prime}$ is the same as $B M$.

- Coordinates of p. $M^{\prime}: x_{M^{\prime}}=\frac{K_{A M} \cdot x_{A^{\prime}}-K_{B M} \cdot x_{B^{\prime}}+y_{B^{\prime}}-y_{A^{\prime}}}{K_{A M}-k_{B M}} ; y_{M^{\prime}}=y_{B^{\prime}}+K_{B M} \cdot x_{M^{\prime}}-x_{B^{\prime}}$

- Length of the segment $O M^{\prime}: O M^{\prime}=\sqrt{x_{M^{\prime}}^{2}+y_{M^{\prime}}^{2}}$

\subsection{Tertiary model}

- Length of the segment $A A^{\prime \prime}: A A^{\prime \prime}=\frac{\varepsilon_{1}}{\omega_{1}^{2}} \cdot O A$

- Coordinates of p. C": $x_{C^{\prime \prime}}=0 \quad y_{C^{\prime \prime}}=0$

- Coordinates of p. $A^{\prime \prime}: \quad x_{A^{\prime \prime}}=x_{A}+A A^{\prime \prime} \cdot \cos \left(\frac{\pi}{2}-\varphi\right) \quad y_{A^{\prime \prime}}=y_{A}-A A^{\prime \prime} \cdot \sin \left(\frac{\pi}{2}-\varphi\right)$

IRITIE Vol. 4, No. 3, 2016 ISSN 1314-8788 (print), ISSN 1314-8796 (online), doi: 10.15547/artte.2016.03.004 


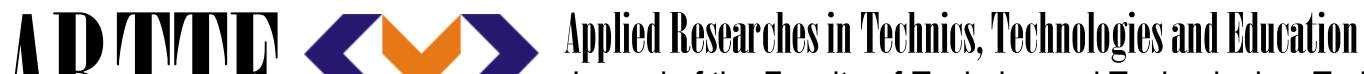 Journal of the Faculty of Technics and Technologies, Trakia University https://sites.google.com/a/trakia-uni.bg/artte/}

- Length of the segment $A^{\prime \prime} N_{1}: A^{\prime \prime} N_{1}=\frac{\left(A^{\prime} B^{\prime}\right)^{2}}{A B}$

- Length of the segment $O N_{2}: O N_{2}=\frac{\left(O B^{\prime}\right)^{2}}{C B}$

- Coordinates of points $N_{1}$ и $N_{2}$

$x_{N 1}=x_{A^{n}}+A^{n} N_{1} \cdot \cos \xi \quad y_{N 1}=y_{A^{n}}+A^{n} N_{1} \cdot \sin \xi$

$x_{N 2}=O N_{2} \cdot \cos \psi \quad y_{N 2}=O N_{2} \cdot \sin \psi$

- Coordinates of p. B":

$$
x_{B^{\prime \prime}}=\frac{\left(\frac{1}{k_{A B}} \cdot x_{N 1}-\frac{1}{k_{B C}} \cdot x_{N 2}\right)-y_{N 2}+y_{N 1}}{\frac{1}{k_{A B}}-\frac{1}{k_{B C}}} y_{B^{\prime \prime}}=y_{N 1}+\frac{1}{k_{A B}} \cdot\left(x_{N 1}-x_{B^{\prime \prime}}\right)
$$

- Slope of the lines, determined by segments $A " B ", A " M "$ u $B " M "$ :

$$
K_{A^{\prime \prime} B^{\prime \prime}}=\frac{y_{A^{\prime \prime}}-y_{B^{\prime \prime}}}{x_{A^{\prime \prime}}-x_{B^{\prime \prime}}} \quad K_{A^{\prime \prime} M^{\prime \prime}}=\frac{k_{A^{\prime \prime} B^{\prime \prime}}+\tan \alpha}{1-k_{A^{\prime \prime} B^{\prime \prime}} \cdot \tan \alpha} \quad K_{B^{\prime \prime} M^{\prime \prime}}=\frac{k_{A^{\prime \prime} B^{\prime \prime}}-\tan \beta}{1+k_{A^{\prime \prime} B^{n}} \cdot \tan \beta}
$$

- Coordinates of p. $M^{\prime \prime}$ :

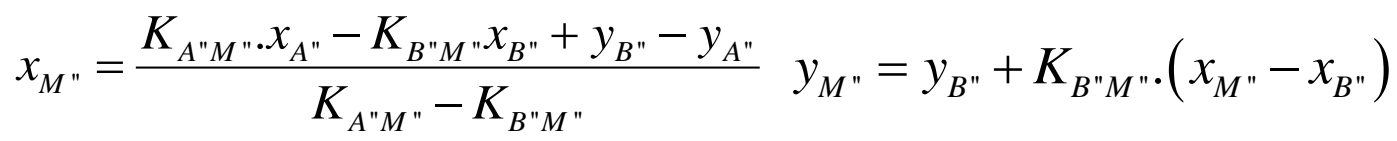

- Length of the segment $O D^{\prime \prime}: O D^{\prime \prime}=\sqrt{x_{D^{\prime \prime}}^{2}+y_{D^{\prime \prime}}^{2}}$

\section{CONCLUSIONS}

This article provides mathematical apparatus that maintains the advantages of "method of models" and avoids voluminous graphics work.

Presented equations are simple and can be implemented in a computer program. Mathematical apparatus allows studying mechanism for a desired number of positions.

\section{REFERENCES}

[1] Piperkov D. Secondary modeling in theoretical mechanics, Ph.D. Dissertation, TU Sofia, 1987.

[2] Tenchev Vasko. A small treatise of velocities and accelerations of planar linkages (second and third-level models), Sofia, 2009. 